\title{
PALIACIÓN EN DIÁLISIS
}

Lic. Carmen Benavides de Díaz*, Mónica Paola Lima González**, Yolanda Paola Ojeda Vargas**, Gloria Angélica Vera Becerra**.

\section{Resumen}

Con el fin de elaborar una gúa de cuidados paliativos en enfermería para el paciente con insuficiencia renal crónica terminal en diálisis y sin posibilidad de transplante, se indagaron los conocimientos que tenían los enfermeros que laboran en diferentes unidades renales de Bogotá, sobre los cuidados paliativos que brindaban a los pacientes, y cómo estos los recibían y aplicaban en su propio cuidado para paliar su enfermedad. Esta información fue obtenida por medio de la aplicación de dos cuestionarios semiestructurados que arrojaron un interés general de mejorar la calidad de vida de los pacientes, a través de cuidados sencillos y efectivos que permiten cumplir tal objetivo. Después la información se complementó con la experiencia de la investigadora y la literatura obtenida acerca del tema en los diferentes textos consultados, todo ello con el propósito de agregar nuevos cuidados y optimizar los ya sugeridos por la población en estudio. Luego se elaboró la guía según los patrones funcionales de M. Gordon y los diagnósticos de la Asociación norteamericana de diagnósticos de enfermería, así como las características definitorias, resultados esperados y los cuidados obtenidos durante la investigación con fundamentos científicos.

Palabras claves: Cuidados de enfermería paliativos, diálisis.

\section{Introducción}

Como resultado del proceso de investigación sobrelos cuidados de enfermería en la paliación en diálisis se presenta la guía, teniendo en cuenta que el cuidado se considera como la esencia de la enfermería, y este a su vez debe estar fundamentado en el conocimiento social, humano, ético, científico y técnico, dándole validez profesional a la ejecución del mismo.

Cuando se hace referencia a los cuidados específicos que el enfermero debe prestar al paciente en la terapia dialítica, es necesario actuar con tacto y seguridad, hablar y obrar con acierto, realizar las actividades con suavidad, dulzura, lentitud moderada y sutil, en forma dúctil y apacible, es decir tener la capacidad de cuidar y enseñar, considerando al paciente merecedor de toda la atención.

La diálisis es la terapia paliativa, indicada en la insuficiencia renal crónica terminal (IRCT) y su procedimiento es invasor, requiere de alta tecnología y complejidad, demandando del profesional de enfermería conocimientos

* Enfermera Especialista en Nefrología Urología, Profesora Asistente Facultad de Enfermería, Fundación Universitaria de Ciencias de la Salud. Investigadora principal.

** Estudiantes de enfermería VII semestre Fundación Universitaria de Ciencias de la Salud científicos tecnológicos y habilidades manuales, adquiridos con la experiencia, así como una gran dosis de compromiso social, humano y ético, que va en pro de la calidad de vida, promueve el bienestar del paciente y acompaña a la familia.

En la revisión bibliográfica nacional e internacional de enfermería no se encontraron registros sobre los cuidados específicos de paliación en diálisis, relacionados con el alivio de signos y síntomas como la sed, el aliento urémico, el prurito, el dolor osteomuscular y la desesperanza que en esta guía se han explicitado. Sin embargo, a través de los cuestionarios realizados como instrumento de recolección de datos a los enfermeros y pacientes, del análisis teórico y la experiencia del investigador, se pudieron identificar los cuidados de enfermería paliativos para estas manifestaciones clínicas propias de la uremia, enmarcados en los patrones funcionales de Margory Gordon y los diagnósticos de enfermería de la Asociación norteamericana de diagnósticos de enfermería (NANDA) 2002.

\section{Materiales y métodos}

Siguiendo los objetivos específicos determinados para este trabajo, se inició con la revisión bibliográfica relativa al tema de paliación aplicada a la diálisis, en lo referente 
al manejo de algunos de los signos y síntomas propios de la uremia.

En segunda instancia, se elaboró una encuesta para enfermeros con experiencia mayor de dos años en la atención de pacientes en diálisis, en donde se preguntó cuáles son los cuidados que brindan y enseñan a los pacientes cuando presentan aumento de peso por alta ingesta de líquidos, sed, prurito en la piel, dolor osteomuscular, desesperanza y así mismo se indagó qué hace cuando aparecen estas manifestaciones clínicas. A través de cuantificación se analizó la recolección de datos y análisis de la información de forma numérica, pues las respuestas se clasificaron por frecuencia y porcentaje. La cualificación se basa en las experiencias tanto de las enfermeras como de los pacientes ante la necesidad de paliar un síntoma.

Una vez reunida la información el grupo investigador analizó los cuidados, los relacionó y agrupó teniendo en cuenta los patrones de M. Gordon y los diagnósticos de enfermería de la NANDA, obteniendo así las bases para la elaboración de la guía.

\section{Resultados}

Al analizar los datos obtenidos en el instrumento aplicado tanto a enfermeros como a pacientes, se encontró que la gran mayoría de pacientes no cumplen con el control de líquidos establecidos, en tanto que el $70 \%$ de los enfermeros optan por brindar educación con el propósito de concienciar al paciente sobre la importancia de seguir el régimen dietario.

Con respecto al cuidado de la piel, las enfermeras sugieren el uso de cremas humectantes y el cumplimiento de la terapia dialítica entre otros, ya que esto contribuye a disminuir los síntomas de la uremia.

Dentro de la información obtenida por los pacientes para el manejo del dolor por osteodistrofia, son muy pocos los recursos empleados por ellos diferentes al analgésico prescrito; por su parte, el enfermero posee conocimientos sobre diferentes recursos útiles para el usuario, como por ejemplo masajes, ejercicios y otros que merecen ser compartidos con sus pacientes para que estos dispongan de estrategias que contribuyan a mejorar su calidad de vida con respecto al dolor.
Referente al sueño y descanso en el paciente renal, se evidencia que muy pocos ven la actividad física y el ejercicio entre sus aficiones como alternativas útiles para dormir y descansar adecuadamente en la noche.

Desde el punto de vista emocional, los pacientes renales son los más lábiles, debido a la sintomatología que presentan y los cambios que se ven obligados a realizar en su estilo de vida, llevándolos a experimentar sentimientos que van desde la irritabilidad y apatía, hasta la depresión y desesperanza.

Según los patrones funcionales de M.Gordon y los diagnósticos de enfermería de NANDA, se organizaron en la guía los cuidados paliativos de enfermería en diálisis, necesarios para el paciente con IRCT sin posibilidad de transplante.

\section{Conclusiones}

- Gracias a la experiencia obtenida en la investigación, se sustrae la afirmación de que el enfermero posee los conocimientos sobre como aliviar los síntomas pero no lo titulan bajo el nombre de paliación, sus cuidados lo realizan intuitivamente como parte del proceso de atención de enfermería, ya que la paliación va enfocada a mitigar el sufrimiento, como morir dignamente y aspectos relacionados con la defunción.

- La educación es un aspecto importante, tanto para el paciente como para el enfermero. La actualización contribuye al mejoramiento de la calidad de vida de estos enfermos. Cuando el enfermero imparte educación continua sobre los cuidados de la sintomatología, los pacientes reflejan esta instrucción en su propio cuidado. Si ésta es esporádica o no se da, los pacientes toman otras opciones.

- Los enfermeros explicitan cuidados para la piel, el control de líquidos, el manejo de la sed y el alivio del dolor; alternativas de gran diversidad para paliar al paciente con IRCT que no se encuentran escritas en los libros. Esto contribuye a mejorar la calidad de vida y aumentar el acervo de conocimientos para las enfermeras que están en entrenamiento para el manejo de estos pacientes y a los usuarios que realizan la terapia dialítica. 
- Los líquidos, la piel y el estreñimiento son aspectos en los cuales el enfermero puede intervenir como actividades independientes de enfermería; sin embargo, hay otros aspectos interdisciplinarios como la calidad de diálisis y el tratamiento farmacológico, que no se desligan del manejo de estos síntomas.

\section{Bibliografía consultada}

1. Gómez C. Cuidados Significado y Sentido. Coloquio Nacional de Enfermería.

2. DoyleD H. Texto de medicina paliativa. Universidad de Oxfort; 1993.p 8

3. Guía práctica de la clínica. Bogotá: Instituto de Seguro Social basada en la evidencia científica. Insuficiencia Renal Crónica. p. 26-27.

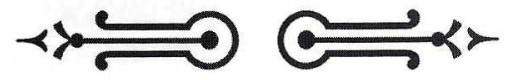

